\title{
Effect of stretching on interfacial stability
}

\author{
C. Pozrikidis, San Diego, California, and M. G. Blyth, Norwich, England \\ Received September 18, 2003; revised March 3, 2004 \\ Published online: May 24, 2004 (C) Springer-Verlag 2004
}

\begin{abstract}
Summary. The effect of stretching on the stability of a horizontal interface between two fluids with different viscosities and densities is discussed. A local elongational flow acts to reduce the amplitude of perturbations and increase the wavelength of periodic waves, and thereby alter the instantaneous growth rate of disturbances. Linear stability analysis for Stokes flow reveals that, in the case of a horizontal interface between two semi-infinite fluids subjected to orthogonal stagnation-point flow, interfacial stretching is not able to suppress the Rayleigh-Taylor instability of unstably stratified fluids. In contrast, stretching is able to suppress the growth of periodic waves on the surface of a flat film resting on a horizontal surface. Numerical simulations based on the boundary-integral method for Stokes flow confirm that localized perturbations on the film surface are suppressed when the elongational flow is sufficiently strong.
\end{abstract}

\section{Introduction}

The effect of an elongational flow on the evolution of localized disturbances and periodic waves at the interface between two fluids is twofold. First, the acceleration of the fluid in the plane of the interface increases the lateral extent of the disturbances and the wave length of periodic waves. Second, because the elongational flow stretches the interface, the accompanying fluid deceleration normal to the interface reduces the amplitude of perturbations. While the second effect has an obvious stabilizing influence, the significance of the first effect on the transient and long-time behavior of perturbations depends on the particular application.

Consider, for example, the stability of an infinite cylindrical liquid thread of radius $a$, suspended in another ambient fluid. In the absence of fluid motion, the column is susceptible to the Rayleigh capillary instability promoting the growth of interfacial waves with wave length $L$ and wave number $k \equiv 2 \pi / L$, provided that $k a<1$; when $k a>1$, the waves oscillate undamped in inviscid flow, and decay in viscous flow (e.g., [8]). The effect of an ambient axisymmetric stagnation-point flow was discussed by Tomotika [10], Mikami et al. [4], and more recently by Kwak et al. [2], in the limit of vanishing Reynolds numbers. Because both the thread radius and the wave number of periodic waves decrease during the evolution, the reduced wave number $k a$ eventually enters the unstable regime $k a<1$, and the perturbation becomes unstable. Consequently, the behavior of periodic waves at long times depends on the asymptotic behavior of the growth rate in the limit $k a \rightarrow 0$. A formal stability analysis shows that, unless the viscosity of the thread or ambient fluid is exactly equal to zero, the perturbation is eventually stabilized by interfacial stretching.

In this article, we discuss a different type of interface stabilization due to stretching, related to the Rayleigh-Taylor instability of a stationary or accelerated interface. This flow configuration occurs, for example, at the top of a gas bubble rising through an ambient liquid. The main goal 
is to resolve whether an orthogonal stagnation-point flow, and more generally an elongational flow, is able to suppress the growth of periodic waves in the limit of vanishing Reynolds numbers.

In Sect. 2, we consider the stability of the interface between two semi-infinite fluids separated by a horizontal interface, and formulate the linear stability problem for periodic perturbations. The results will show that the elongational flow is unable to suppress the Rayleigh-Taylor instability due to an unstable density stratification. In Sect. 3, we consider the stability of the interface between a liquid film resting on a horizontal wall underneath an overlying semiinfinite fluid. In this case, the unperturbed film is continuously thinning under the influence of the elongational flow, though the film thickness remains uniform in the absence of perturbations. The results will show that interfacial stretching eventually suppresses the growth of interfacial perturbations. To describe the finite-amplitude motion, the linear stability analysis is complemented by numerical simulations based on a boundary-element method for Stokes flow. Results on the evolution of localized perturbations will confirm that the elongational flow indeed has a stabilizing influence by washing away interfacial corrugations.

\section{Rayleigh-Taylor instability of a planar interface}

We consider a semi-infinite fluid with viscosity $\mu_{1}$ and density $\rho_{1}$, lying underneath another semi-infinite fluid with viscosity $\mu_{2}$ and density $\rho_{2}$, as shown in Fig. 1. An orthogonal stagnation-point flow with a generally time-dependent strength is imposed on either side of the interface. In the unperturbed configuration the interface coincides with the horizontal streamline passing through the stagnation point. Sufficiently close to the stagnation point viscous stresses dominate, and the motion of the fluid is governed by the linear equations of Stokes flow,

$\mathbf{0}=-\nabla p+\mu \nabla^{2} \mathbf{u}+\rho \mathbf{g}, \quad \nabla \cdot \mathbf{u}=0$,

where $\mathbf{u}=(u, v)$ is the velocity, $p$ is the pressure, $\mu$ is the viscosity, $\rho$ is the density, and $\mathbf{g}$ is the acceleration of gravity. The $x$ - and $y$-velocity components are given by

$u_{j}^{(0)}=G(t) x, \quad v_{j}^{(0)}=-G(t) y$,

for $j=1,2$, corresponding to the lower and upper fluid, where $G(t)$ is the strength of the imposed elongational flow, and the superscript $(0)$ denotes the unperturbed configuration.

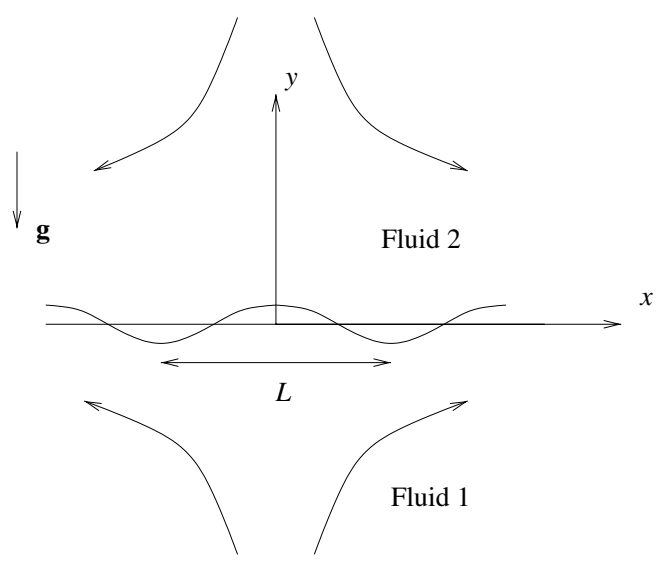

Fig. 1. Illustration of a planar interface separating two semi-infinite fluids in the presence of an orthogonal stagnation-point flow 
The associated pressure field is given by

$p_{1}^{(0)}=-\rho_{1} g y+P_{0} . \quad p_{2}^{(0)}=-\rho_{2} g y+2 \mu_{1}(1-\lambda) G(t)+P_{0}$,

where $\lambda=\mu_{2} / \mu_{1}$ is the viscosity ratio, and $P_{0}$ is an unspecified constant.

The preceding expressions for the velocity and pressure may readily be confirmed to satisfy the usual conditions of continuity of velocity and surface traction at the interface. It is worth noting that the pressure undergoes a jump to compensate differences in the viscous normal stress due to the different fluid viscosities. The Stokes flow solution ceases to be valid when one of the Reynolds numbers, $R e \equiv G(t) x_{i} x_{j} \rho_{k} / \mu_{k}$, where $x_{1}=x, x_{2}=y$, and $i, j, k=1,2$, is no longer sufficiently small. Having noted this limitation, we proceed to investigate the stability of the basic configuration working under the auspices of Stokes flow.

\subsection{Linear stability analysis}

In the stability analysis, we consider two-dimensional spatially periodic perturbations with time-dependent wave length $L(t)$ and wave number $k(t)=2 \pi / L(t)$, and describe the position of the interface as

$y=f(x, t)=\epsilon \eta(x, t)$,

where $\epsilon$ is a dimensionless parameter whose magnitude is much less than unity,

$\eta(x, t)=A_{1}(t) \cos [k(t) x]$

is the wave form of the perturbation, and $A_{1}(t)$ is the amplitude of the interface. Since the wave length evolves according to $\mathrm{d} L / \mathrm{d} t=G(t) L$, the wave number $k(t)$ evolves according to

$\frac{\mathrm{d} k}{\mathrm{~d} t}=-G(t) k$.

The linearity of the equations of Stokes flow allows us to introduce an evolution equation in the form of the first-order differential equation

$\frac{\mathrm{d} A_{1}}{\mathrm{~d} t}=\sigma(t) A_{1}$,

where $\sigma(t)$ is the instantaneous growth rate.

Two special cases are of particular interest. First, when the fluid densities and viscosities are matched and the surface tension is zero, the interface is advected passively with the flow, and its amplitude evolves according to the equation $\mathrm{d} A_{1} / \mathrm{d} t=\left(\partial v^{(0)} / \partial y\right)_{y=0} A_{1}=-G(t) A_{1}$, corresponding to the growth rate

$\sigma(t)=-G(t)$.

Second, in the absence of a stagnation-point flow, $G=0$, a normal-mode stability analysis provides us with the constant growth rate (e.g., [6])

$\sigma=\frac{1}{2 \mu_{1}(1+\lambda)}\left[\frac{\left(\rho_{2}-\rho_{1}\right) g}{k}-k \gamma\right]$.

A seemingly approximate growth rate applicable under more general circumstances can be derived by combining these two special cases, obtaining

$\sigma(t)=\frac{1}{2 \mu_{1}(1+\lambda)}\left[\frac{\left(\rho_{2}-\rho_{1}\right) g}{k(t)}-k(t) \gamma\right]-G(t)$. 
In the case of unstable stratification, $\rho_{2}>\rho_{1}$, the first term in the square brackets dominates when the wave number becomes sufficiently small. Thus, even though a wave may decay during a transient period due to the combined effect of interfacial stretching and surface tension, it will eventually start growing due to the Rayleigh-Taylor instability. Consequently, the stretching of the interface is unable to suppress the instability at long times.

To put the preceding arguments on a more rigorous basis, we perform a formal linear stability analysis. We begin by expressing the perturbation stream functions corresponding to the displacement (2.4) in the form of the periodic biharmonic functions

$\psi_{j}^{(1)}(x, y, t)=\phi_{j}(y, t) \sin [k(t) x]$,

where the superscript (1) denotes the perturbation. By definition, the perturbation $x$ - and $y$ velocity components are given by $u_{j}^{(1)}=\partial \psi_{j}^{(1)} / \partial y$ and $v_{j}^{(1)}=-\partial \psi_{j}^{(1)} / \partial x$. Requiring that the perturbation decays far from the interface, we find

$\phi_{1}(y, t)=a_{1}(t) \mathrm{e}^{\hat{y}}+b_{1}(t) \hat{y} \mathrm{e}^{\hat{y}}, \quad \phi_{2}(y, t)=c_{2}(t) \mathrm{e}^{-\hat{y}}+d_{2}(t) \hat{y} \mathrm{e}^{-\hat{y}}$,

where $\hat{y}=k y$, and $a_{1}(t), b_{1}(t), c_{2}(t), d_{2}(t)$, are four real functions to be determined by satisfying the linearized interfacial conditions. The corresponding perturbation pressure fields can be expressed in the form

$p_{j}^{(1)}(x, y, t)=\mu_{j} q_{j}(y, t) \cos [k(t) x]$,

for $j=1,2$. Substituting the preceding expressions in the $x$-component of the Stokes equation and rearranging, we find

$q_{j}(y, t)=k \frac{\partial \phi_{j}}{\partial y}-\frac{1}{k} \frac{\partial^{3} \phi_{j}}{\partial y^{3}}$.

Kinematic compatibility requires $D(f-y) / D t=0$ at the interface, where $D / D t$ is the material derivative. In the linearized approximation,

$\frac{\partial \eta}{\partial t}+\left.u^{(0)}\right|_{y=0} \frac{\partial \eta}{\partial x}-\left(\frac{\partial v^{(0)}}{\partial y}\right)_{y=0} \eta-\left.v^{(1)}\right|_{y=0}=0$.

Substituting the preceding expressions and using (2.6) to simplify, we derive the evolution equation

$\frac{\mathrm{d} A_{1}}{\mathrm{~d} t}=-G(t) A_{1}-k(t) a_{1}$.

Next, we consider the linearized kinematic and dynamic interface conditions. Continuity of the $x$ - and $y$-velocity components requires, respectively,

$$
\begin{aligned}
\left(\frac{\partial u_{1}^{(0)}}{\partial y}\right)_{y=0} \eta(x, t)+\left(\frac{\partial \psi_{1}^{(1)}}{\partial y}\right)_{y=0} & =\left(\frac{\partial u_{2}^{(0)}}{\partial y}\right)_{y=0} \eta(x, t)+\left(\frac{\partial \psi_{2}^{(1)}}{\partial y}\right)_{y=0}, \\
\psi_{1}^{(1)}(x, y=0, t) & =\psi_{2}^{(1)}(x, y=0, t) .
\end{aligned}
$$

Substituting the preceding expressions for the stream function, we find

$a_{1}+b_{1}=-c_{2}+d_{2}, \quad a_{1}=c_{2}$.

The $x$ - and $y$-components of the interfacial force balance require, respectively,

$\mu_{1}\left(\frac{\partial u_{1}^{(1)}}{\partial y}+\frac{\partial v_{1}^{(1)}}{\partial x}\right)_{y=0}-\mu_{2}\left(\frac{\partial u_{2}^{(1)}}{\partial y}+\frac{\partial v_{2}^{(1)}}{\partial x}\right)_{y=0}=4 G \mu_{1}(1-\lambda) \frac{\partial \eta}{\partial x}$, 
and

$$
\left(-p_{1}^{(1)}+2 \mu_{1} \frac{\partial v_{1}^{(1)}}{\partial y}\right)_{y=0}-\left(-p_{2}^{(1)}+2 \mu_{2} \frac{\partial v_{2}^{(1)}}{\partial y}\right)_{y=0}+\left(\rho_{1}-\rho_{2}\right) g \eta(x)=\gamma \frac{\partial^{2} \eta}{\partial x^{2}} .
$$

Substituting the preceding expressions in (2.19), we find

$a_{1}+b_{1}-\lambda\left(c_{2}-d_{2}\right)=-2(1-\lambda) \frac{G}{k} A_{1}$.

Using Eq. (2.18.1) to simplify the left-hand side of (2.21), we obtain

$a_{1}+b_{1}=\xi A_{1}$,

where we have defined

$\xi \equiv-2 \frac{1-\lambda}{1+\lambda} \frac{G}{k}$.

Condition (2.20) reduces to

$$
\left(-\mu_{1} q_{1}+\mu_{2} q_{2}-2 k \mu_{1}(1-\lambda) \frac{\partial \phi_{1}}{\partial y}\right)_{y=0}=-\left[\left(\rho_{1}-\rho_{2}\right) g+\gamma k^{2}\right] A_{1} .
$$

Substituting $q_{1}(y=0)=-2 k^{2} b_{1}, q_{2}(y=0)=-2 k^{2} d_{2}$, and $\left(\partial \phi_{1} / \partial y\right)_{y=0}=k\left(a_{1}+b_{1}\right)=k \xi A_{1}$, we obtain

$2 k^{2} \mu_{1}\left[b_{1}-\lambda d_{2}-(1-\lambda) \xi A_{1}\right]=-\left[\left(\rho_{1}-\rho_{2}\right) g+\gamma k^{2}\right] A_{1}$,

which may be rearranged into

$b_{1}-\lambda d_{2}=\chi A_{1}$,

where

$\chi \equiv(1-\lambda) \xi-\Psi$,

and

$\Psi \equiv \frac{\left(\rho_{1}-\rho_{2}\right) g+\gamma k^{2}}{2 k^{2} \mu_{1}}$.

Finally, we combine Eqs. (2.18) to obtain $2 a_{1}+b_{1}-d_{2}=0$, and compile equations (2.22) and (2.26) to formulate the linear system

$$
\left[\begin{array}{ccc}
2 & 1 & -1 \\
1 & 1 & 0 \\
0 & 1 & -\lambda
\end{array}\right]\left[\begin{array}{l}
a_{1} \\
b_{1} \\
d_{2}
\end{array}\right]=\left[\begin{array}{l}
0 \\
\xi \\
\chi
\end{array}\right] A_{1}
$$

Solving this system by elementary methods, we find $a_{1}=\Psi /(1+\lambda)$, which can be substituted in (2.16) to yield the growth rate stated in (2.10), without any approximation. Thus, the instantaneous growth rate depends linearly on the instantaneous rate of extension, independent of the fluid viscosities.

Interestingly, the solution of the linear system (2.29) for the stream function coefficients $b_{1}$ and $d_{2}$ depends on both $\xi$ and $\Psi$, and so does the disturbance flow accompanying the periodic perturbation. Specifically, we find $b_{1}=\xi-\Psi /(1+\lambda)$ and $d_{2}=-[(1-2 \lambda) \xi-2 \Psi] /(1+\lambda)$. As expected, when $\lambda=1, \xi=0$, a disturbance flow in response to the elongational flow does not arise. 


\section{Rayleigh-Taylor instability of a liquid film on a plane wall}

Consider now a liquid film with viscosity $\mu_{1}$ and density $\rho_{1}$, resting on a horizontal wall underneath another semi-infinite fluid with viscosity $\mu_{2}$ and density $\rho_{2}$, as shown in Fig. 2. An orthogonal stagnation-point flow with a generally time-dependent strength is imposed far from the wall. In the unperturbed configuration, the interface is flat, and the film thickness decreases in time under the action of the extensional flow. In the vicinity of the stagnation point, the fluid motion is governed by the equations of Stokes flow describing quasi-steady evolution.

In a stationary frame of reference temporarily positioned at the instantaneous location of the flat interface, $y=0$, as shown in Fig. 2, the $x$ - and $y$-velocity components and pressure inside the film are given by

$$
\begin{aligned}
& u_{1}^{(0)}=2 G(t) x(y+h), \quad v_{1}^{(0)}=-G(t)(y+h)^{2}, \\
& p_{1}^{(0)}=-\rho_{1} g y-2 \mu_{1} G(t) y+P_{0},
\end{aligned}
$$

where $G(t)$ is the strength of the imposed elongational flow, $h(t)$ is the instantaneous film thickness, and $P_{0}$ is an unspecified pressure. The velocity and pressure in the upper semi-infinite fluid are given by the companion expressions

$$
\begin{aligned}
& u_{2}^{(0)}=2 G(t) x\left(\frac{y}{\lambda}+h\right), \quad v_{2}^{(0)}=-G(t)\left(\frac{y^{2}}{\lambda}+2 y h+h^{2}\right), \\
& p_{2}^{(0)}=-\rho_{2} g y-2 \mu_{1} G(t) y+4 \mu_{1}(1-\lambda) G(t) h+P_{0}
\end{aligned}
$$

where $\lambda=\mu_{2} / \mu_{1}$ is the viscosity ratio. The preceding expressions satisfy the conditions of continuity of velocity and stress at the interface, as well as the no-slip and no-penetration boundary conditions at the wall, located at $y=-h(t)$. When $\lambda=1$, the two velocity and pressure fields combine into a unified form expressing orthogonal stagnation-point flow toward a plane wall, derived by Moffatt [5]. The film thickness decreases in time according to the kinematic condition

$\frac{\mathrm{d} h}{\mathrm{~d} t}=v(y=0)=-G(t) h^{2}$

which can be integrated to give

$h(t)=\frac{h_{0}}{1+h_{0} \int_{0}^{t} G\left(t^{\prime}\right) \mathrm{d} t^{\prime}}$,

where $h_{0} \equiv h(t=0)$.

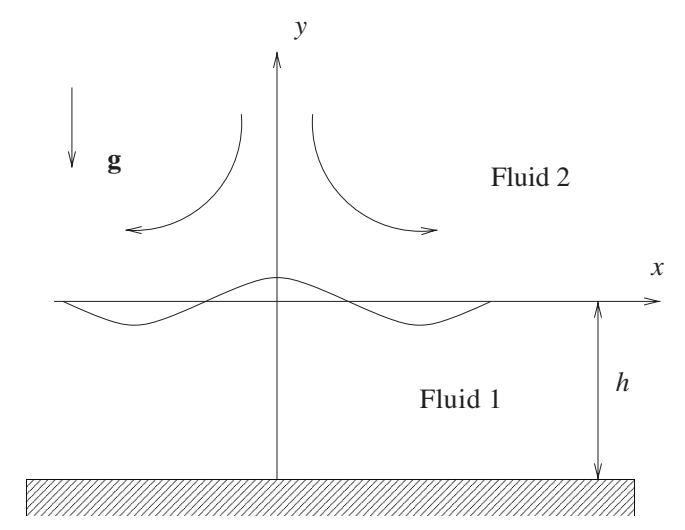

Fig. 2. Illustration of a liquid film resting on a horizontal wall underneath a semi-infinite fluid, subject to an orthogonal stagnation-point flow 


\subsection{Linear stability analysis}

To study the stability of the planar interface subject to periodic perturbations, we describe the position of the interface as shown in (2.4) and (2.5). The amplitude of the interface evolves according to (2.7), where $\sigma$ is the growth rate. As in the case of semi-infinite fluids discussed in Sect. 2, two extreme cases are of special interest. First, when the viscosities and densities of the fluids are matched and the surface tension vanishes, the interface is advected passively with the flow, and its amplitude evolves according to $\mathrm{d} A_{1} / \mathrm{d} t=(\partial v / \partial y)_{y=0} A_{1}=-2 G(t) h(t) A_{1}$, yielding $\sigma(t)=-2 G(t) h(t)$.

Second, in the absence of a stagnation-point flow, $G=0$, a normal-mode stability analysis provides us with the constant growth rate [6]

$\sigma=\frac{1}{2 \mu_{1}(1+\lambda)}\left[\frac{\left(\rho_{2}-\rho_{1}\right) g}{k}-\gamma k\right] \Phi(\hat{k}, \lambda)$,

where $\hat{k}=k h$ and

$\Phi(\hat{k}, \lambda)=(1+\lambda) \frac{\frac{1}{2} \sinh (2 \hat{k})-\hat{k}+\lambda\left(\sinh ^{2} \hat{k}-\hat{k}^{2}\right)}{\left(1-\lambda^{2}\right) \hat{k}^{2}+(\cosh \hat{k}+\lambda \sinh \hat{k})^{2}}$.

In the limit of short waves, $\hat{k} \rightarrow \infty$, the function $\Phi$ tends to unity, yielding the growth rate of perturbations on the interface between two semi-infinite fluids stated in (2.9). Graphs of the function $\Phi$ for several values of $\lambda$ are shown in Fig. 3.

A potentially acceptable and apparently approximate growth rate applicable under more general circumstances can be derived by combining these two special cases, obtaining

$\sigma(t) \simeq \frac{1}{2 \mu_{1}(1+\lambda)}\left[\frac{\left(\rho_{2}-\rho_{1}\right) g}{k(t)}-\gamma k(t)\right] \Phi(\hat{k}, \lambda)-2 G(t) h(t)$.

In the limit of long waves, $\hat{k} \rightarrow 0, \Phi \rightarrow \frac{2}{3}(1+\lambda) \hat{k}^{3}$, yielding the asymptotic expansion

$\sigma \simeq\left[\frac{g\left(\rho_{2}-\rho_{1}\right)}{3 \mu_{1}} \hat{k}^{2}-2 G(t)\right] h(t)$.

To appreciate the significance of this estimate, we consider an unstable stratification, $\rho_{2}>\rho_{1}$. Since, in the course of the evolution, the first term inside the square brackets in (3.9) becomes decreasingly small, the growth rate will eventually become negative manifesting decay, unless the strength of the elongational flow, $G(t)$, is also reduced at an appropriate rate. If $G$ is

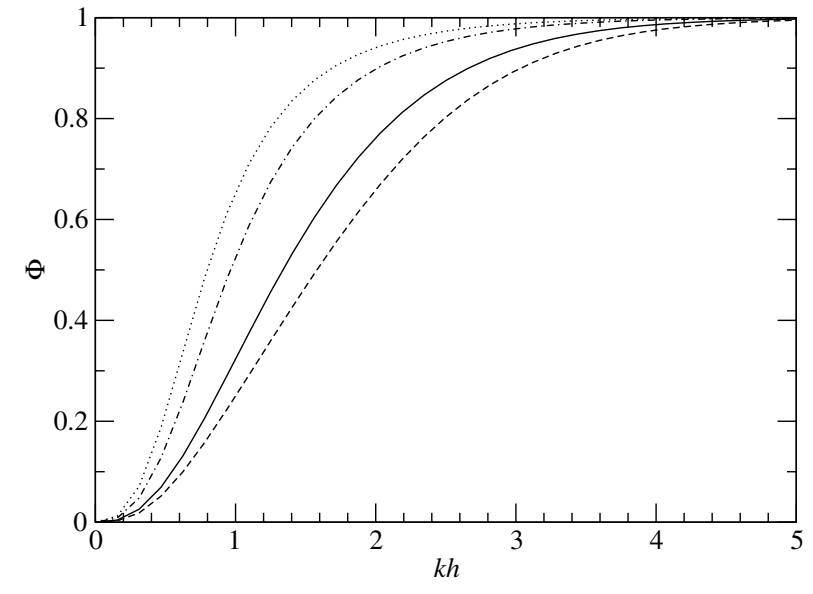

Fig. 3. Graphs of the modulating function $\Phi$ determining the effect of a wall on the Rayleigh-Taylor instability of liquid film for viscosity ratio $\lambda=0.1$ (dashed line), 1.0 (solid line), 5.0 (dotdashed line), and 10.0 (dotted line) 
held constant, even though the waves may grow during a transient period, they will eventually be stabilized due to stretching.

\subsection{Linear stability analysis}

In the linear stability analysis, we consider periodic perturbations in the interfacial shape described by (2.4) and (2.5). Working in hindsight, we express the perturbation streamfunctions by the non-periodic and even with respect to $x$-forms

$\psi_{j}^{(1)}(x, y, t)=\phi_{j}(y, t) \sin [k(t) x]+\hat{x} \chi_{j}(y, t) \cos [k(t) x]$,

where $\hat{x}=k y$ and $\hat{y}=k y$. Requiring that the stream functions satisfy the biharmonic equation and demanding that the perturbation velocity decays as $y \rightarrow \infty$, we find

$$
\begin{aligned}
\phi_{1}(y, t)= & a_{1}(t) \mathrm{e}^{\hat{y}}+b_{1}(t) \hat{y} \mathrm{e}^{\hat{y}}+c_{1}(t) \mathrm{e}^{-\hat{y}}+d_{1}(t) \hat{y} \mathrm{e}^{-\hat{y}} \\
& +b_{1}^{\prime}(t)\left(\frac{3}{2}-2 \hat{y}+\hat{y}^{2}\right) \mathrm{e}^{\hat{y}}-d_{1}^{\prime}(t)\left(\frac{3}{2}+2 \hat{y}+\hat{y}^{2}\right) \mathrm{e}^{-\hat{y}}, \\
\chi_{1}(y, t)= & a_{1}^{\prime}(t) \mathrm{e}^{\hat{y}}+b_{1}^{\prime}(t) \hat{y} \mathrm{e}^{\hat{y}}+c_{1}^{\prime}(t) \mathrm{e}^{-\hat{y}}+d_{1}^{\prime}(t) \hat{y} \mathrm{e}^{-\hat{y}},
\end{aligned}
$$

for the flow inside the film, and

$\phi_{2}(y, t)=c_{2}(t) \mathrm{e}^{-\hat{y}}+d_{2}(t) \hat{y} \mathrm{e}^{-\hat{y}}-d_{2}^{\prime}(t)\left(\frac{3}{2}+2 \hat{y}+\hat{y}^{2}\right) \mathrm{e}^{-\hat{y}}$,

$\chi_{2}(y, t)=c_{2}^{\prime}(t) \mathrm{e}^{-\hat{y}}+d_{2}^{\prime}(t) \hat{y} \mathrm{e}^{-\hat{y}}$,

for the flow in the semi-infinite fluid, where $a_{j}(t), b_{j}(t), \ldots$, are real functions. The corresponding perturbation pressure fields can be expressed in the form

$p_{j}^{(1)}(x, y, t)=\mu_{j}\left(q_{j}(y, t) \cos [k(t) x]-\hat{x} r_{j}(y, t) \sin [k(t) x]\right)$,

for $j=1,2$. Substituting the preceding expressions in the $x$-component of the Stokes equation and rearranging, we find

$$
\begin{aligned}
& q_{j}(y, t)=k \frac{\partial \phi_{j}}{\partial y}-\frac{1}{k} \frac{\partial^{3} \phi_{j}}{\partial y^{3}}+k \frac{\partial \chi_{j}}{\partial y}+\frac{1}{k} \frac{\partial^{3} \chi_{j}}{\partial y^{3}}, \\
& r_{j}(y, t)=k \frac{\partial \chi_{j}}{\partial y}-\frac{1}{k} \frac{\partial^{3} \chi_{j}}{\partial y^{3}} .
\end{aligned}
$$

Kinematic compatibility requires $D(h+f-y) / D t=0$ at the interface, where $D / D t$ is the material derivative. At leading order we recover (3.3), and at first order we obtain (2.15). Substituting the preceding expressions in (2.15), we derive an evolution equation for the wave number,

$\frac{\mathrm{d} k}{\mathrm{~d} t}=-2 G(t) \hat{k}-k^{2} \frac{c_{2}^{\prime}}{A_{1}}$

and a companion evolution equation for the perturbation amplitude,

$\frac{\mathrm{d} A_{1}}{\mathrm{~d} t}+2 G h A_{1}=-k\left[\phi_{2}(0)+\chi_{2}(0)\right]=-k\left(c_{2}-\frac{3}{2} d_{2}^{\prime}+c_{2}^{\prime}\right)$.

Thus, the growth rate of the perturbation is given by

$\sigma(t)=-\frac{k}{A_{1}}\left(c_{2}-\frac{3}{2} d_{2}^{\prime}+c_{2}^{\prime}\right)-2 G(t) h(t)$. 
Continuity of the $x$-velocity component at the position of the perturbed interface requires the satisfaction of the condition (2.17.1). Upon evaluation, we find

$a_{1}+b_{1}-c_{1}+d_{1}-\frac{1}{2}\left(b_{1}^{\prime}+d_{1}^{\prime}\right)=-c_{2}+d_{2}-\frac{1}{2} d_{2}^{\prime}$,

and

$a_{1}^{\prime}+b_{1}^{\prime}-c_{1}^{\prime}+d_{1}^{\prime}+c_{2}^{\prime}-d_{2}^{\prime}=\frac{2 G}{k^{2}} A_{1}\left(\frac{1}{\lambda}-1\right)$.

Continuity of the $y$ velocity component at the position of the perturbed interface requires the satisfaction of condition (2.17.2). Upon evaluation, we find

$a_{1}+c_{1}+\frac{3}{2}\left(b_{1}^{\prime}-d_{1}^{\prime}\right)=c_{2}-\frac{3}{2} d_{2}^{\prime}, \quad a_{1}^{\prime}+c_{1}^{\prime}=c_{2}^{\prime}$.

The $x$-component of the interfacial force balance requires

$\mu_{1}\left(\frac{\partial u_{1}^{(1)}}{\partial y}+\frac{\partial v_{1}^{(1)}}{\partial x}\right)_{y=0}-\mu_{2}\left(\frac{\partial u_{2}^{(1)}}{\partial y}+\frac{\partial v_{2}^{(1)}}{\partial x}\right)_{y=0}=8 G h \mu_{1}(1-\lambda) \frac{\partial \eta}{\partial x}$.

Substituting the preceding expressions, we find

$a_{1}+b_{1}+c_{1}-d_{1}-\lambda\left(c_{2}-d_{2}\right)-\frac{1}{2} c_{2}^{\prime}(1-\lambda)=-4(1-\lambda) \frac{G h}{k} A_{1}$,

$b_{1}^{\prime}-d_{1}^{\prime}+c_{2}^{\prime}(1-\lambda)+\lambda d_{2}^{\prime}=0$.

The $y$-component of the interfacial force balance requires condition (2.20). Substituting the preceding expressions, we find

$\left[\left(\rho_{1}-\rho_{2}\right) g+k^{2} \gamma\right] A_{1}=k^{2} \mu_{1}\left[3\left(b_{1}^{\prime}+d_{1}^{\prime}-\lambda d_{2}^{\prime}\right)+2\left(a_{1}-c_{1}+\lambda c_{2}\right)\right]$,

$a_{1}^{\prime}-c_{1}^{\prime}+\lambda c_{2}^{\prime}=0$.

The no-slip and no-penetration conditions at the wall require

$\phi_{1}=\chi_{1}=0, \quad \frac{\partial \phi_{1}}{\partial y}=\frac{\partial \chi_{1}}{\partial y}=0$

at $y=-h$, providing us with four further scalar equations for the unknown constants. These may be appended to conditions (3.18), (3.19), (3.20), (3.21), and (3.22), and the boundary and interfacial conditions can be assembled into the matrix form

$\mathbf{M} \cdot \mathbf{w}=\mathbf{b}$,

where $\mathbf{M}$ is a $12 \times 12$ matrix of coefficients, the solution vector is

$\mathbf{w}=\left(a_{1}, b_{1}, c_{1}, d_{1}, c_{2}, d_{2}, a_{1}^{\prime}, b_{1}^{\prime}, c_{1}^{\prime}, d_{1}^{\prime}, c_{2}^{\prime}, d_{2}^{\prime}\right)^{T}$,

and $\mathbf{b}$ includes the inhomogeneous parts of the interfacial and wall conditions. The linear system (3.25) was solved using the symbolic manipulation package Maple, and the growth rate was then computed using (3.17).

To compare the exact numerical results with the approximate prediction (3.6), we express the growth rate in the form

$\sigma=\frac{1}{2 \mu_{1}(1+\lambda)}\left[\frac{\left(\rho_{2}-\rho_{1}\right) g}{k}-\gamma k\right] \Phi(\hat{k}, \lambda)-2 G(t) h(t) Q(\hat{k}, \lambda)$, 
where $Q$ is a dimensionless function given by

$Q=\frac{(1+\lambda)^{4} e^{4 \hat{k}}+4 \alpha_{1} e^{2 \hat{k}}+2 \alpha_{2}+4 \alpha_{3} e^{-2 \hat{k}}+(1-\lambda)^{4} e^{-4 \hat{k}}}{\left[(1+\lambda)^{2} e^{2 \hat{k}}+2\left(1-\lambda^{2}\right)\left(1+2 \hat{k}^{2}\right)+(1-\lambda)^{2} e^{-2 \hat{k}}\right]^{2}}$,

and the constants $\alpha_{1}$ and $\alpha_{2}$ are given by

$$
\begin{aligned}
& \alpha_{1}=(1-\lambda)(1+\lambda)^{2}[(1+\lambda)+2 \hat{k}(1+(\lambda-1) \hat{k})], \\
& \alpha_{2}=(1-\lambda)^{2}(1+\lambda)\left[3(1+\lambda)+8 \hat{k}\left[1+\hat{k} \lambda+\hat{k}^{3}(\lambda-1)\right]\right], \\
& \alpha_{3}=(1-\lambda)^{3}\left[2 \hat{k}+(1+\lambda)\left(1+2 \hat{k}^{2}\right)\right] .
\end{aligned}
$$

Figure 4 shows graphs of $Q$ plotted against $\hat{k}$ for several values of $\lambda$. When $\lambda=1, Q$ is uniformly equal to unity for any reduced wave number $\hat{k}$, in agreement with our earlier discussion. In the limit $\hat{k} \rightarrow 0$ or $\infty, Q$ tends to unity for any viscosity ratio, conforming with (3.6). Thus, in the limit of either small or large wave number disturbances, the growth rate coincides with the approximate prediction. These results lend credence to the conclusions of the approximate analysis in Sect. 3.1 concerning the stabilizing influence of the stagnation-point flow.

\subsection{Numerical simulations}

To provide further insights into the stability of the thinning film, we consider the evolution of localized perturbations centered at the stagnation point of the incident flow, and simulate the motion using the boundary-integral formulation for Stokes flow.

To develop the integral formulation, we decompose the flow into the undisturbed basic component described by (3.1) and (3.2), denoted by the superscript $B$, and a disturbance component denoted by the superscript $D$, so that $\mathbf{u}=\mathbf{u}^{B}+\mathbf{u}^{D^{(j)}}$, for $j=1,2$, where the superscripts (1) and (2) denote, respectively, the film and overlying fluid. Working in a standard way [7], we derive an integral equation of the second kind for the interfacial distribution of the perturbation velocity of the lower fluid, $\mathbf{u}^{D^{(1)}}$. Specifically, we find that the perturbation velocity at the point $\mathbf{x}_{0}$ that lies at the interface, denoted by $I$, is given by the integral representation

$$
\begin{aligned}
\mathbf{u}^{D^{(1)}}\left(\mathbf{x}_{0}\right)= & -\frac{\lambda}{1+\lambda} \Delta \mathbf{u}^{B}\left(\mathbf{x}_{0}\right)-\frac{2}{1+\lambda} \mathbf{I}^{S L P}\left(\mathbf{x}_{0}, \Delta \mathbf{f}^{D}\right) \\
& +2 \frac{1-\lambda}{1+\lambda} \check{\mathbf{I}}^{D L P}\left(\mathbf{x}_{0}, \mathbf{u}^{D^{(1)}}\right)-2 \frac{\lambda}{1+\lambda} \check{\mathbf{I}}^{D L P}\left(\mathbf{x}_{0}, \Delta \mathbf{u}^{B}\right),
\end{aligned}
$$

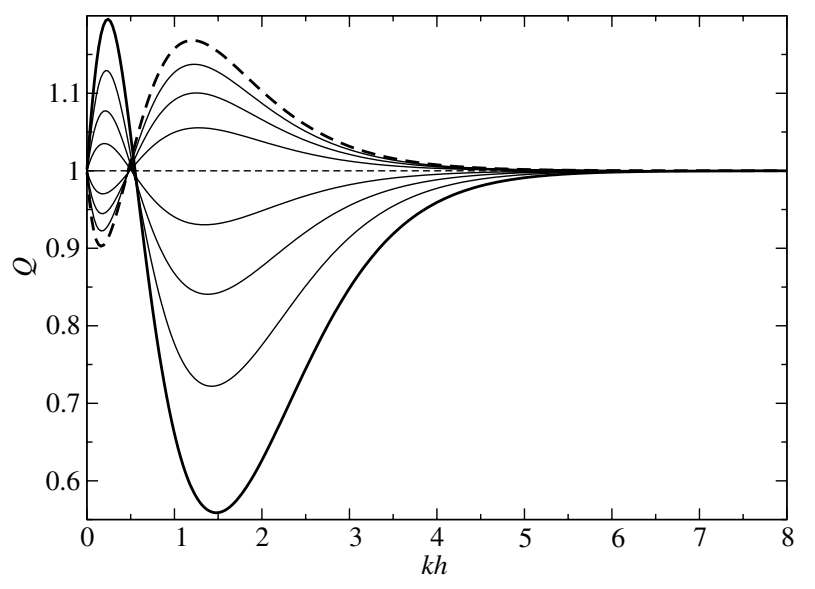

Fig. 4. Graphs of the modulating function $Q(\hat{k}, \lambda)$ for $\lambda=0.2$ (heavy solid line) up to $\lambda=1.8$ (heavy dashed line) plotted in equal intervals of 0.2 . The light dashed line describing $Q=1$ corresponds to $\lambda=1.0$ 
where $\Delta \mathbf{u}^{B} \equiv \mathbf{u}^{B^{(1)}}-\mathbf{u}^{B^{(2)}}$ is the jump in the velocity of the basic flow across the interface. The $x$ - and $y$-components of this jump are given by

$\Delta u^{B}=2\left(1-\frac{1}{\lambda}\right) G x y, \quad \Delta v^{B}=-\left(1-\frac{1}{\lambda}\right) G y^{2}$.

To simplify the notation, we have denoted the single-layer Stokes flow potential by

$I_{j}^{S L P}\left(\mathbf{x}_{0}, \mathbf{f}\right) \equiv \frac{1}{4 \pi \mu_{1}} \int_{I}^{P V} G_{i j}\left(\mathbf{x}, \mathbf{x}_{0}\right) f_{i}(\mathbf{x}) \mathrm{d} l(\mathbf{x})$,

and the principal value (PV) of the double-layer Stokes flow potential by

$\check{I}_{j}^{D L P}\left(\mathbf{x}_{0}, \mathbf{u}\right) \equiv \frac{1}{4 \pi} \int_{I}^{P V} u_{i}(\mathbf{x}) T_{i j k}\left(\mathbf{x}, \mathbf{x}_{0}\right) n_{k}(\mathbf{x}) \mathrm{d} l(\mathbf{x})$,

where $G_{i j}$ is the velocity Green's function of two-dimensional Stokes flow representing the flow induced by a point force above a plane wall, and $T_{i j k}$ is the associated stress tensor. The strength density of the single-layer potential in the integral representation, $\Delta \mathbf{f}^{D} \equiv\left(\boldsymbol{\sigma}^{D^{(1)}}-\boldsymbol{\sigma}^{D^{(2)}}\right) \cdot \mathbf{n}$, is the jump in the traction of the disturbance flow across the interface, where $\boldsymbol{\sigma}$ is the stress tensor, and $\mathbf{n}$ is the unit normal vector pointing into the film. The dynamic condition at the interface requires

$\Delta \mathbf{f}^{D} \equiv \Delta \mathbf{f}-\Delta \mathbf{f}^{B}=\gamma \kappa \mathbf{n}-\Delta \mathbf{f}^{B}$,

where $\kappa$ is the curvature of the interface in the $x y$-plane, reckoned to be positive when the interface is upward parabolic. Finally, the jump in the traction of the basic flow across the interface is given by

$\Delta \mathbf{f}^{B}=\left(\rho_{1}-\rho_{2}\right) g y \mathbf{n}+8 \mu_{1} G h(1-\lambda)\left[\begin{array}{c}n_{x} \\ 0\end{array}\right]$,

where $n_{x}$ is the $x$ component of the unit normal vector.

To solve the integral equation, we divide a truncated portion of the interface into $N$ boundary elements, and assume that all functions defined over the interface take constant values over the individual elements, yielding the discrete representation

$$
\begin{aligned}
u_{j}^{D^{(1)}}\left(\mathbf{x}_{0}\right)= & -\frac{\lambda}{1+\lambda} \Delta u_{j}^{B}\left(\mathbf{x}_{0}\right)-\frac{2}{1+\lambda} \sum_{m=1}^{N} A_{i j m}\left(\mathbf{x}_{0}\right)\left[\Delta f_{i}^{D}\right]_{m} \\
& +2 \frac{1-\lambda}{1+\lambda} \sum_{m=1}^{N} B_{i j m}\left(\mathbf{x}_{0}\right)\left[u_{i}^{D^{(1)}}\right]_{m}-2 \frac{\lambda}{1+\lambda} \sum_{m=1}^{N} B_{i j m}\left(\mathbf{x}_{0}\right)\left[\Delta u_{i}^{B}\right]_{m},
\end{aligned}
$$

where $[\cdot]_{m}$ denotes the $m$-th element quantity, and summation over $i=1,2$ is implied on the right-hand side. We have introduced the influence coefficients

$A_{i j m}\left(\mathbf{x}_{0}\right) \equiv \frac{1}{4 \pi \mu_{1}} \int_{E_{m}} G_{i j}\left(\mathbf{x}, \mathbf{x}_{0}\right) \mathrm{d} l(\mathbf{x})$

and

$B_{i j m}\left(\mathbf{x}_{0}\right) \equiv \frac{1}{4 \pi} \int_{E_{m}}^{P V} T_{i j k}\left(\mathbf{x}, \mathbf{x}_{0}\right) n_{k}(\mathbf{x}) \mathrm{d} l(\mathbf{x})$,

where $E_{m}$ denotes the $m$-th element.

Positioning the point $\mathbf{x}_{0}$ at the middle of the $q$-th boundary element, $\mathbf{x}_{q}^{M}$, and rearranging, we recast (3.35) into the form 


$$
\begin{aligned}
& \sum_{m=1}^{N}\left[\delta_{q m} \delta_{i j}-2 \frac{1-\lambda}{1+\lambda} B_{i j m}\left(\mathbf{x}_{q}^{M}\right)\right]\left[u_{i}^{D^{(1)}}\right]_{m}=-\frac{2}{1+\lambda} \sum_{m=1}^{N} A_{i j m}\left(\mathbf{x}_{q}^{M}\right)\left[\Delta f_{i}^{D}\right]_{m} \\
& -\frac{\lambda}{1+\lambda} \sum_{m=1}^{N}\left[\delta_{q m} \delta_{i j}+2 B_{i j m}\left(\mathbf{x}_{q}^{M}\right)\right]\left[\Delta u_{i}^{R}\right]_{m}
\end{aligned}
$$

where $\delta_{q m}$ and $\delta_{i j}$ are Kronecker's deltas. The linear system encapsulated in (3.38) for $q=1, \ldots, N$ is solved by Gauss elimination, and the marker points defining the boundary elements are advected in time using the second-order Runge-Kutta method. The normal vector and curvature are computed by clamped cubic-spline interpolation (e.g., [9]).

In the case of constant strength $G$, the evolution of a perturbation depends on the dimensionless rate of extension and capillary number defined, respectively, by

$\hat{G} \equiv \frac{\mu_{1} G}{\left(\rho_{2}-\rho_{1}\right) g}, \quad C a \equiv \frac{\mu_{1} G h_{0}^{2}}{\gamma}$,

where $h_{0}$ is the unperturbed layer thickness at the origin of time. Additional parameters include the viscosity ratio, $\lambda$, and the initial shape of the interface. In the simulations presented in this section, the initial shape is described by

$y(x, t=0)=h_{0}\left[1+\epsilon \cos \pi \hat{x} \exp \left(-\frac{\hat{x}^{2}}{4}\right)\right]$,

where $\hat{x} \equiv x / h_{0}$, and $\epsilon$ is a specified amplitude. The solution domain of the integral equation is truncated at the distance $L= \pm 10 h_{0}$ on either side of the stagnation point. To demonstrate the stabilizing action of the elongational flow, the surface tension was set to zero in all computations, corresponding to $C a=\infty$. For future reference, we introduce the dimensionless time $t^{\prime} \equiv t \mu /\left(\Delta \rho g h_{0}\right)$, where $\Delta \rho=\rho_{2}-\rho_{1}$.

Figure 5 shows stages in the evolution of the interface for $\lambda=0.1,1$, and 10 , and perturbation amplitude $\epsilon=0.20$, in the absence of a stagnation-point flow, $\hat{G}=0$. Because the upper fluid is heavier than the lower fluid, $\rho_{2}>\rho_{1}$, the interface falls prey to the Rayleigh-Taylor instability, which causes interfacial amplification and the eventual development of rising plumes. These results are consistent with the earlier simulations of Newhouse and Pozrikidis [6] for periodic perturbations. We find, in particular, that the viscosity ratio plays an important role in determining the evolving interfacial morphology.
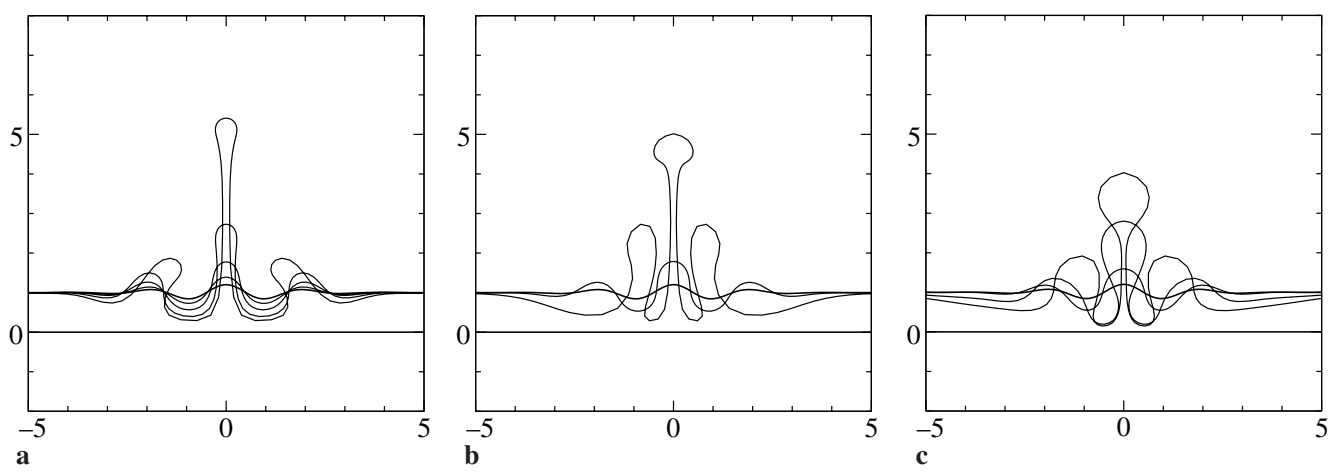

Fig. 5. Rayleigh-Taylor instability of unstably stratified fluids in the absence of a stagnation-point flow, $\hat{G}=0$. Interfacial profiles for a $\lambda=0.1$ (high film viscosity) at $t^{\prime}=0,5,10,15,20 ; \mathbf{b} \lambda=1$, at $t^{\prime}=0,20,40,50 ; \mathbf{c} \lambda=10$ (low film viscosity) at $t^{\prime}=0,80,160,200$. The initial profiles are drawn with heavy lines 


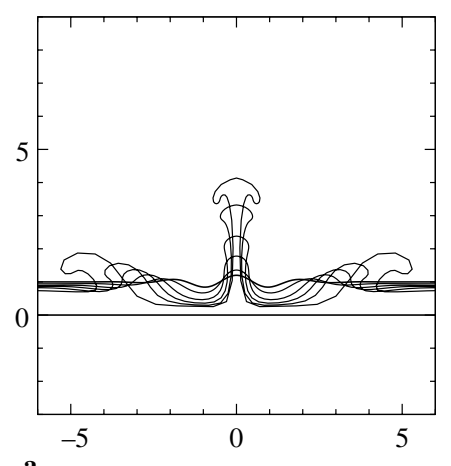

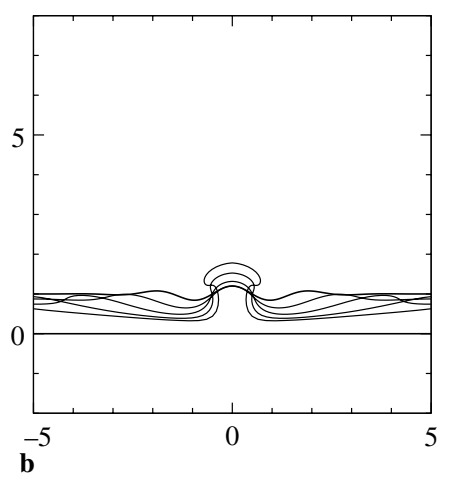

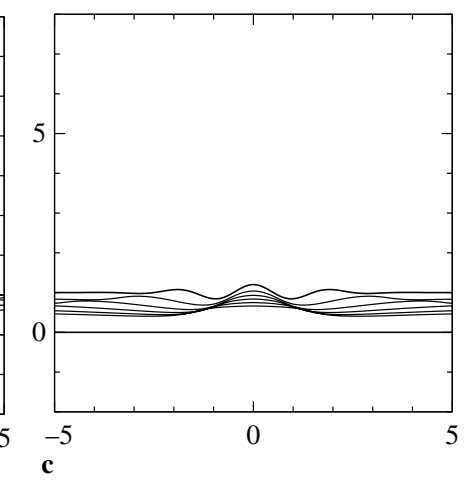

Fig. 6. Suppression of the Rayleigh-Taylor instability for $\lambda=0.1$. Interfacial profiles for $\mathbf{a} \hat{G}=0.01$ at $t^{\prime}=0,6,12,16,20,26 ; \mathbf{b} \hat{G}=0.03$ at $t^{\prime}=0,5,10,15,20 ; \mathbf{c} \hat{G}=0.1$. at $t^{\prime}=0,2,4,6,8,10$. The initial profiles are drawn with heavy lines

Figure 6 demonstrates the effect of the stagnation-point flow for $\lambda=0.1$. The three panels shown in this figure extend the results shown in Fig. 5a for an increasingly strong stagnationpoint flow. The results confirm that the Rayleigh-Taylor instability is suppressed when $\hat{G}$ is sufficiently high. In particular, interfacial perturbations are washed away giving rise to overturning and cusped waves. The smaller the initial amplitude of the perturbation, the lower the value of $\hat{G}$ that stabilizes the interface. Similar results are obtained for different viscosity ratios.

\section{Discussion}

We have discussed the behavior of periodic waves on stretched interfaces in infinite and wallbounded flow, and discovered that interfacial stretching can have a temporary or permanent stabilizing influence on the Rayleigh-Taylor instability. Because the stability analysis was conducted under the auspices of Stokes flow, inertial modes present in single-fluid flow at nonzero Reynolds numbers do not arise. These inertial modes complement the interfacial modes presently considered.

In the case of single-fluid flow against a plane wall, the Stokes-flow approximation is valid near (and is the inner solution of) the stagnation point of the inclusive Hiemenz orthogonal stagnation-point flow, which is applicable for any Reynolds number. In the case of flow against a film resting on a plane wall discussed in Sect. 3, the Stokes-flow solution is valid as long as the thickness of the Hiemenz boundary layer is greater than the instantaneous film thickness.

In the case of homogeneous stagnation-point flow, stability analyses have been conducted by previous authors on the assumption that the perturbation stream function takes the nonperiodic separable form $\psi \sim x^{m} F(y) \exp (\sigma t)$, where $m$ is an arbitrary exponent set equal to the Görtler value of unity in most theoretical studies, and the function $F(y)$ is required to decay exponentially as $y \rightarrow \infty$ (e.g., [1], [3]). It is possible that this class of disturbances is also relevant to the case of the film flow, though the unbounded growth of the film thickness along the wall hinders their physical interpretation in applications.

\section{Acknowledgements}

This research was supported by a grant provided by the National Science Foundation. 


\section{References}

[1] Brattkus, K., Davis, S. H.: The linear stability of plane stagnation-point flow against general disturbances. Q. J. Mech. Appl. Math. 44, 135-146 (1991).

[2] Kwak, S., Fyrillas, M. M., Pozrikidis, C.: Effect of surfactants on the instability of a liquid thread, Part II: Extensional flow. Int. J. Multiphase Flow 27, 39-60 (2001).

[3] Lasseigne, D. G., Jackson, T. L.: Stability of a nonorthogonal stagnation flow to threedimensional disturbances. Theor. Comput. Fluid Dyn. 3, 207-218 (1992).

[4] Mikami, T., Cox, R. G., Mason, S. G.: Breakup of extending liquid threads. Int. J. Multiphase Flow 2, 113-138 (1975).

[5] Moffatt, H. K.: Viscous and resistive eddies near a sharp corner. J. Fluid Mech. 18, 1-18 (1964).

[6] Newhouse, L. A., Pozrikidis, C.: The Rayleigh-Taylor instability of a liquid layer resting on a plane wall. J. Fluid Mech. 217, 615-638 (1990).

[7] Pozrikidis, C.: Boundary integral and singularity methods for linearized viscous flow. Cambridge: Cambridge University Press 1992.

[8] Pozrikidis, C.: Introduction to theoretical and computational fluid dynamics. New York: Oxford University Press 1997.

[9] Pozrikidis, C.: Numerical computation in science and engineering. New York: Oxford University Press 1998.

[10] Tomotika, S.: Breaking up of a drop of viscous liquid immersed in another viscous fluid which is extending at a uniform rate. Proc. Roy. Soc. A 153, 302-318 (1936).

Authors' addresses: C. Pozrikidis, Department of Mechanical and Aerospace Engineering, University of California, San Diego, La Jolla, CA 92093-0411, U.S.A. (E-mail: cpozrikidis@ucsd.edu); M. G. Blyth, School of Mathematics, University of East Anglia, Norwich, NR4 7TJ, U.K. (E-mail: m.blyth@uea.ac.uk) 